\title{
Magnetic Characterization of Melt-Spun Co-Ni-Ga Ferromagnetic Superelastic Alloy
}

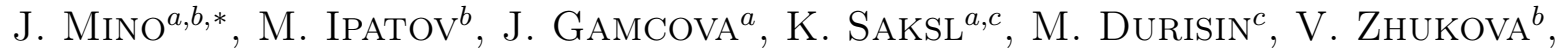 \\ Z. VARGOVA ${ }^{a}$, A. ZHUKOV ${ }^{b, d, e}$ AND R. VARGA ${ }^{a}$ \\ ${ }^{a}$ Institute of Physics, Faculty of Sciences, P.J. Safarik University, Park Angelinum 9, 04154 Košice, Slovakia \\ ${ }^{b}$ Material Physics Department, Faculty of Chemistry, University of the Basque Country, UPV/EHU, \\ Plaza Elhuyar, 2, 20018, San Sebastian, Spain \\ ${ }^{c}$ Institute of Material Research, SAS, Watsonova 47, 04001 Košice, Slovakia \\ ${ }^{d}$ IKERBASQUE, Basque Foundation for Science, 48011 Bilbao, Spain \\ ${ }^{e}$ Dpto. de Física Aplicada, EUPDS, UPV/EHU, 200018, San Sebastian, Spain
}

\begin{abstract}
Ribbons of composition $\mathrm{Co}_{49} \mathrm{Ni}_{21} \mathrm{Ga}_{30}$ have been prepared by melt-spinning method. X-ray diffraction investigation revealed single phase with $B 2$ structure at room temperature. However, analysis of magnetization dependence of temperature suggests phase transition in the range 150-250 K. Resistivity measurements revealed similar transition with shift to higher temperatures in the presence of magnetic field.
\end{abstract}

DOI: 10.12693/APhysPolA.131.1075

PACS/topics: $62.20 . \mathrm{fg}$

\section{Introduction}

Ferromagnetic superelastic alloys (FSA) have attracted scientific attention in recent years, since they are perspective materials that can be used as sensors and actuators [1]. Properties of superelastic change are mostly influenced by martensitic transformation (MT), which appears between two solid phases called martensite and austenite by coordinated shear relocation of atoms over very short distances (usually in order of $\AA$ ). It can be triggered by a stress, by a temperature and, in the case of the ferromagnetic alloys [2], by a magnetic field change. Thus, ferromagnetic superelastic materials have advantage in having one more control parameter. Austenite is high temperature phase and it is stable without stress. Martensite is low temperature phase and it is stabilized by stress. Variations between crystal structures of both phases and their lattice parameters result in strain. It is known from studies on single crystals that we can achieve maximum strain in one direction that is strictly determined by shape of both changing crystal structures [3] Some applications require preferred crystal orientation, which can be used to achieve maximum strain in defined direction.

So far, many alloys with aforementioned properties have been discovered, for example alloys NiMnGa $[2$ 4], FePd [5], CoNi(Al or Ga) [6-9], FeMnAlNi [10, 11], $\mathrm{Co} 2 \mathrm{CrGaSi}$ [12], or FSA are usually produced by arcmelting $[6,9,11,12]$, by rapid solidification method, or by growing of single crystals $[4,10]$ with additional annealing. However, melt spinning method can help to avoid

*corresponding author; e-mail: jakub.mino@student.upjs.sk long-term annealing (or at least significantly reduce it) keeping good qu ality of highly textured polycrystalline ribbons [13]. So far, there has been successful progress on rapidly quenched ribbons, where only short-term annealing is required to relax the induced internal stress [7].

In this work, we show that rapid quenching method is efficient method for producing a ferromagnetic superelastic alloy that is in a single phase at room temperature and shows phase transformation with different magnetic properties of both phases.

\section{Experimental}

Master alloy with off-stoichiometric composition of the Heusler alloys $\left(\mathrm{Co}_{49} \mathrm{Ni}_{21} \mathrm{Ga}_{30}\right)$ have been produced using arc melting method in an argon atmosphere from highly pure elements (Co - 99.9+\%, Ni - 99.95+\%, Ga 99.99\%). Ribbon have been produced from master alloy using melt spinning technique in a helium atmosphere. Tangential speed of surface of a copper wheel was set to $20 \mathrm{~m} \mathrm{~s}^{-1}$. Scanning electron microscope (SEM) JEOL 6100 operated at $30 \mathrm{kV}$ was used to examine the microstructure of the sample and it was equipped with Xray energy dispersive spectroscopy system (EDX) Inca Energy 200. SEM ran in secondary electrons detection mode. X-ray diffractometer (XRD) RTG Philips Xpert Pro equipped with $\mathrm{Cu}$ anode $\left(K_{\alpha 1}, \lambda=0.154089 \mathrm{~nm}\right)$ was used to study structure of produced ribbons. Magnetic measurements were carried by Quantum Design physical property measurement system (PPMS) and by magnetic properties measurement system (MPMS) in the 5-400 K temperature range under the external magnetic field up to $50 \mathrm{kOe}$. Electric current used in resistivity measurements have been set to $1 \mathrm{~mA}$. Sample was longitudinally oriented to the magnetic field in both measurement systems. 


\section{Result and discussion}

Microstructure analysis provided by SEM revealed polycrystalline structure with a size of crystallites varying between 10 to $30 \mu \mathrm{m}$. Thickness of the ribbon is $30 \mathrm{\mu m}$ (measured from the SEM picture of crosssection shown in Fig. 1). Directional growth of crystallites (perpendicular to ribbon plane) is most probably caused by strong thermal gradient created during rapid quenching. EDX analyses confirmed nominal composition $\mathrm{Co}_{49.4} \mathrm{Ni}_{20.6} \mathrm{Ga}_{30.0}$, which corresponds well with desired one (Fig. 2). Analyses were taken from 5 sites on three different pieces of ribbon and deviation from nominal composition was $\pm 0.8 \%$. Sufficient homogeneity of sample was achieved without long-term annealing of master alloys comparing to [7].

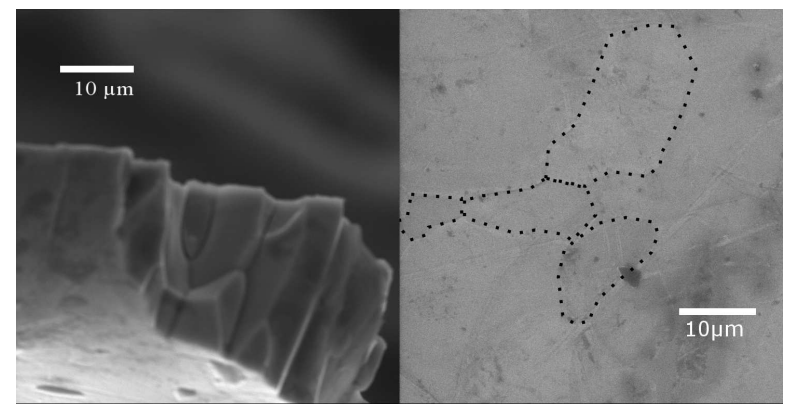

Fig. 1. SEM picture of ribbon cross-section and the surface of ribbon plane with marked grains.

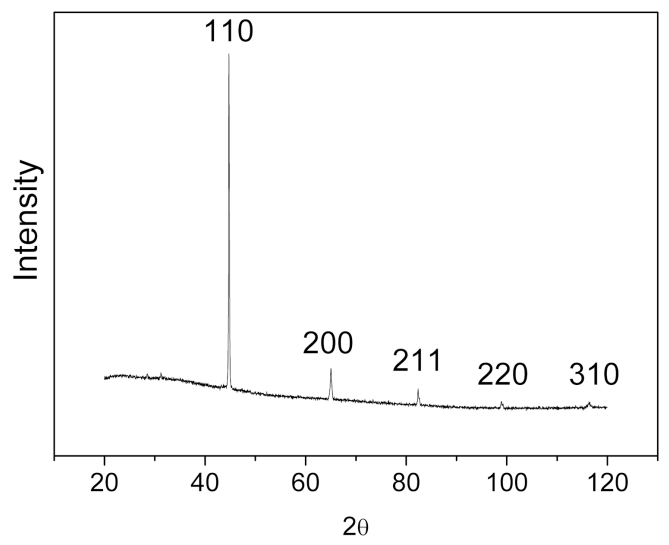

Fig. 2. X-ray diffraction pattern of $\mathrm{Co}_{49} \mathrm{Ni}_{21} \mathrm{Ga}_{30}$ ribbon measured at room temperature.

X-ray diffractogram measured at room temperature shows single phase considered to be $B 2$ crystal structure ( $Y-Z$ disorder of $L 2$ Heusler alloy) [14]. Lattice parameter for the phase was determined to be $2.857 \AA$.

Magnetic characterization revealed a structural transition at the temperature below room temperature. Transformation occurs between high-temperature austenite and low-temperature martensite phase (Fig. 3). XRD of austenite phase corresponds to Ref. [6] and it crystallizes in body centered cubic (bcc) crystal structure, so according to [6] martensite phase should crystallize in face centered tetragonal (fct) structure at low temperature.

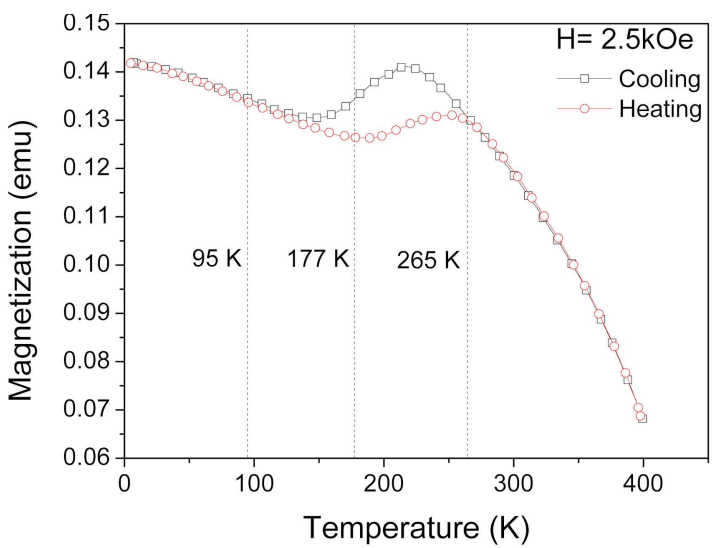

Fig. 3. Magnetization dependence on temperature of $\mathrm{Co}_{49} \mathrm{Ni}_{21} \mathrm{Ga}_{30}$ ribbon determines phase transformation range. Dotted lines represent temperatures used for hysteresis loops measurement.

The phase transformation exhibits a hysteresis, from which MT temperatures were obtained. Austenite start temperature $\left(A_{s}\right)$ was determined to be $179 \mathrm{~K}$ austenite finish temperature $\left(A_{f}\right)$ to be $252 \mathrm{~K}$. Martensite phase transformation starts $\left(M_{s}\right)$ at $216 \mathrm{~K}$ and finish $\left(M_{f}\right)$ at $149 \mathrm{~K}$. The transition of polycrystalline sample is spread into the wilder temperature range contrary to single crystal [8].

Both phases have different saturated magnetization and magnetocrystalline anisotropy, that was confirmed by measurements of hysteresis loops.

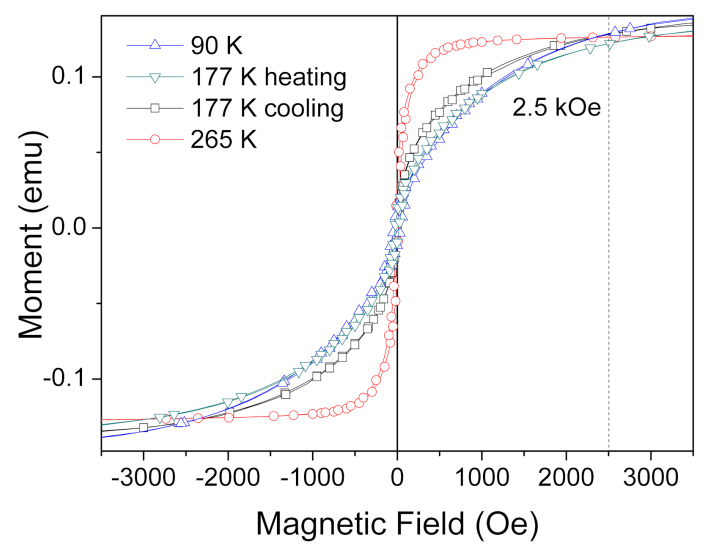

Fig. 4. Hysteresis loops of $\mathrm{Co}_{49} \mathrm{Ni}_{21} \mathrm{Ga}_{30}$ ribbon in austenite, martensite, and in the middle of the MT. Dotted line represents magnetic field used for temperature dependence of magnetization.

Hysteresis loops (shown in Fig. 4) were taken at different temperatures: at full-martensite temperature (90 K), 
twice during the transition $(177 \mathrm{~K})$ on cooling and heating cycle, at full austenite temperature $(265 \mathrm{~K})$. Hysteresis loops indicate that low-temperature phase is more anisotropic.

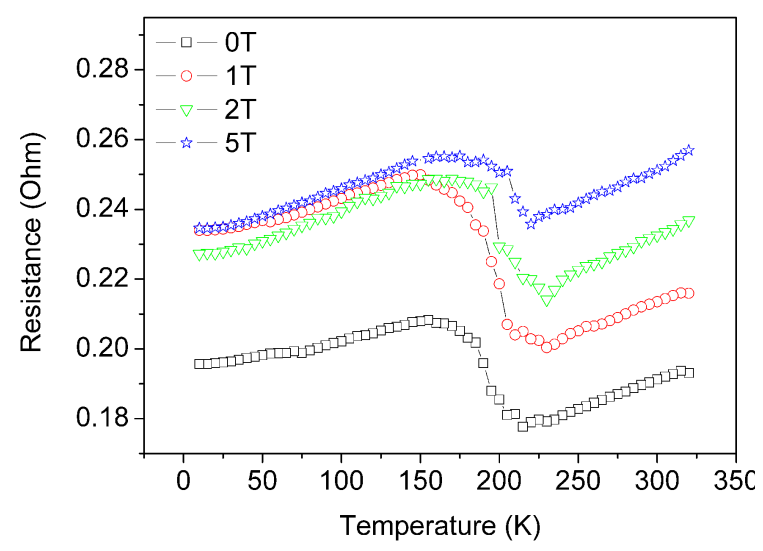

Fig. 5. Temperature dependence of resistance of $\mathrm{Co}_{49} \mathrm{Ni}_{21} \mathrm{Ga}_{30}$ ribbon.

Resistivity dependence on temperature (Fig. 5) confirms MT with $M_{s}=215 \mathrm{~K}$ and for $M_{f}=155 \mathrm{~K}$ in accordance with magnetic measurements. $M_{s}$ and $M_{f}$ dependence on magnetic field does not reveal monotonic increase (Table I), since these temperatures are sensitive to local changes of structure in alloy. On the other side, inflex points of resistivity dependence on temperature (where major part of transition takes place) shows almost monotonic increase with applied magnetic field.

\section{TABLE I}

Martensitic transformation temperature taken from resistivity measurements.

\begin{tabular}{c|c|c|c|c}
\hline \hline & $0 \mathrm{~T}$ & $1 \mathrm{~T}$ & $2 \mathrm{~T}$ & $5 \mathrm{~T}$ \\
\hline martensite start & 215 & 230 & 230 & 220 \\
inflex point & 191 & 200 & 199 & 210 \\
martensite finish & 155 & 151 & 163 & 164
\end{tabular}

\section{Conclusions}

Ferromagnetic superelastic alloy $\mathrm{Co}_{49} \mathrm{Ni}_{21} \mathrm{Ga}_{30}$ has been produced by melt spinning method. The alloy was characterized as single phase with $B 2$ structure. Magnetic measurements revealed phase transformation in temperature range 149-252 K. From resistivity measurement, we can see that it is possible to shift MT temperatures to higher temperatures.

\section{Acknowledgments}

This work was supported by Spanish MINECO under MAT2013-47231-C2-1-P and Slovak project APVV-0027011, VEGA grants No. 1/0164/16, VVGS-PF-2015-495 and VVGS-PF-2016-72614. Technical and human support provided by SGIker is gratefully acknowledged.

\section{References}

[1] J.M. Jani, M. Leary, A. Subic, M.A. Gibson, Mater. Des. , 561078 (2014).

[2] R. Kainuma, Y. Imano, W. Ito, Y. Sutou, H. Morito, S. Okamoto, O. Kitakami, K. Oikawa, A. Fujita, T. Kanomata, K. Ishida, Nature 439, 957 (2006).

[3] V.A. Chernenko, J.M. Barandiarán, V.A. L’vov, J. Gutiérrez, P. Lázpita, I. Orue, J. Alloys Comp. 577, S305 (2013).

[4] V.A. Chernenko, E. Villa, D. Salazar, J.M. Barandiaran, Appl. Phys. Lett. 108, 071903 (2016).

[5] T. Okazaki, Y. Iwai, Y. Furuya, Mater. Trans. 49, 360 (2008).

[6] J. Liu, H. Xie, Y. Huo, H. Zheng, Z. Li, J. Alloys Comp. 420, 145 (2006).

[7] Y. Kishi, C. Criaciunescu, M. Sato, T. Okazaki, Y. Fyruya, M. Wuttig, J. Magn. Magn. Mater. 262, L186 (2003).

[8] V.A. Chernenko, J. Pons, E. Cesari, A.E. Perekos, Mater. Sci. Eng. A 378, 357 (2004).

[9] S. Dilibal, H. Sehitoglu, R.F. Hamilton, H.J. Maier, Y. Chumlyakov, Mater. Sci. Eng. A 528, 2875 (2011).

[10] T. Omori, K. Ando, M. Okano, X. Xu, Y. Tanaka, I. Ohnuma, R. Kainuma, K. Ishida, Science 333, 68 (2011).

[11] P. La Roca, J. Medina, C.E. Sobrero, M. Avalos, J.A. Malarria, A. Baruj, M. Sade, MATEC Web of Conf. 33, 04005 (2015).

[12] X. Xu, T. Omori, M. Nagasako, A. Okubo, R.Y. Umetsu, T. Kanomata, K. Ishida, R. Kainuma, Appl. Phys. Lett. 103, 164104 (2013).

[13] J.L. Sánchez Llamazares, T. Sanchez, J.D. Santos, M.J. Pérez, M.L. Sanchez, B. Hernando, Ll. Escoda, J.J. Suñol, R. Varga, Appl. Phys. Lett. 92, 045002 (2008).

[14] T. Graf, C. Felser, S. Parkin, Progr. Solid State Chem. 39, 1 (2011). 